\title{
Contrast-Enhanced Ultrasound Guided Pleural Biopsy Improves Diagnostic Confidence in Multidisciplinary Diagnosis of Pleural Lesions: A 3- year Prospective Study
}

\section{Wenwen Sun}

Tongji University

Yiming Zhou

Shanghai Pulmonary Hospital Affiliated to School of Medicine

\section{Cong Yang}

Shanghai Pulmonary Hospital Affiliated to School of Medicine

\section{Zhengwei Dong}

Shanghai Pulmonary Hospital Affiliated to School of Medicine

\section{ZheMin Zhang}

Shanghai Pulmonary Hospital Affiliated to School of Medicine

\section{Yin Wang}

Shanghai Pulmonary Hospital Affiliated to School of Medicine

Lin Fan ( $\nabla$ fanlinsj@163.com)

Shanghai Pulmonary Hospital Affiliated to School of Medicine

\section{Research Article}

Keywords: Contrast-enhanced ultrasound, ultrasound guided pleural biopsy, pleural lesion, diagnostic efficiency, Infectious pleural lesion囚Malignant pleural lesion

Posted Date: April 20th, 2021

DOI: https://doi.org/10.21203/rs.3.rs-424800/v1

License: (c) (i) This work is licensed under a Creative Commons Attribution 4.0 International License. Read Full License

Version of Record: A version of this preprint was published at BMC Pulmonary Medicine on July 12th, 2021. See the published version at https://doi.org/10.1186/s12890-021-01583-7. 


\section{Abstract}

Objective: To evaluate the accuracy and safety of contrast-enhanced ultrasound (CEUS) guided biopsy in the diagnosis of pleural lesions.

Method: A prospective study was conducted on patients with undiagnosed pleural lesions. Patients who met the inclusion criteria received pleural biopsy guided by CEUS to obtain specimens, followed by histomathological and etiological examinations. After treatment and follow-up, surgical thoracoscopy was performed for cases of undefinite diagnosis.

Result: A total of 460 patients were finally included. CEUS showed internal necrosis in $72.17 \%$ cases and peripheral obvious vessels $55.43 \%$ cases, both of which were significantly higher than the conventional ultrasound imaged $(P 0.00)$. The diagnostic accuracy through CEUS guided biopsy sampling was $98.91 \%$ (455/460). The etiological diagnostic yield achieved 88.82\% (278/313) in infectious lesions. In 330 cases combined pleural effusion, CEUS guided biopsy increased the diagnostic yield from $60.30 \%$ (199/330) to $98.36 \%(325 / 330)$ in all cases $(P 0.00 \otimes$, from $15.56 \%(14 / 90)$ to $94.44 \%(85 / 90)$ in malignant lesions ( $P 0.00 \bigotimes$ and from $77.08 \% \varangle 185 / 240 \otimes$ to $100 \% \varangle 240 / 240, P 0.00$ ) in infectious lesions. No serious adverse events occurred.

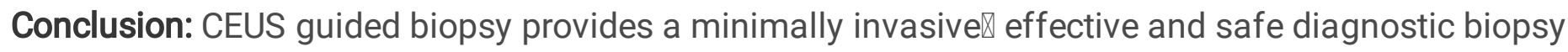
method for pleural lesions.

Clinical Trials Registration: Chinese Clinical Trial Registry ChiCTR2000029749

( ChiCTR, www.chictr.org.cn )

\section{Introduction}

Pleural lesion is a chest imaging manifestation of a variety of diseases $₫$ of which the diagnosis covers multidisciplinary areas regarding surgery[histopathology[microbiology and imaging, etc (1). The best specimen for the diagnosis of pleural disease is pleural tissue rather than pleural effusion (1). Direct sampling from the target lesion is required for microbiology and pathology examinations. Thoracoscopic biopsy has the advantage of direct vision biopsy and is considered to be the most effective sampling method when radiographic evidence of a pleural lesion is present (2). However, it is well known that

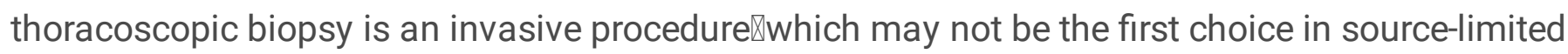
countries, especially in areas with high TB burdens. Ultrasound-guided closed biopsy (USCB) as a minimally invasive method was advocated as a first-line initial diagnostic procedure for undiagnosed pleural effusion, especially in areas with high TB burdens (3-5). However, conventional ultrasound could not better distinguish atelectasis from necrotizing lesions, which often leads to false negative biopsy sampling and result in complications (6). Contrast-enhanced ultrasound (CEUS) is a technology developed on the basis of conventional ultrasound imaging, which can display real-time information of pathological microvascular and blood perfusion. Real-time measurements of tissue microcirculation can 
be performed to differentiate between tissue activity and necrotic lesions then provide the suitable sites for biopsy. In addition, CEUS guided biopsy can conduct real-time guided and accurate targeted puncture biopsy, so as to avoid injury to adjacent organs and large blood vessels, which has the advantages of accurate guidance and fast access to high-quality specimens (7).

Previous study included 58 patients with single pleural lesion to evaluate the diagnostic value of CEUS guided biopsy, obtained an overall diagnostic rate of $98.3 \%$ (8). Our previous study also found the diagnostic efficiency of CEUS guided biopsy in superficial lymph nodes (9). However, there is still a lack of data to verify the effectiveness of this minimally invasive biopsy method in the clinical diagnosis of pleural lesions. Therefore, we conducted a 3-year prospective clinical study to evaluate the value-added effect of CEUS-guided pleural biopsy in the multidisciplinary diagnosis of pleural lesions. The results may provide an alternative minimally invasive diagnostic model for pleural lesions.

\section{Methods}

\section{Ethics Statement and Informed Consent}

The prospective study had been carried out in accordance with the Declaration of Helsinki on ethical principles for the use of human specimens for research. Each patient received written informed consent prior to participation. The study was approved by the Ethics Committee of Shanghai Pulmonary Hospital (approve number K18-170).

\section{Study design and participants[}

This study was conducted in a national pulmonary diseases specialized hospital admitted patients mainly from East China. The ultrasound department of Shanghai Pulmonary Hospital conducts nearly 10000 pleural examinations (ultrasound-guided puncture biopsy囚closed thoracic and abdominal drainage) per year. We prospectively, continuously enrolled patients with pleural lesions.

The inclusion criteria were as follows: Adults ( $\geq 18$ years of age) admitted to the hospital with an undiagnosed pleural lesion requiring biopsy. Eligibility is based on clinical manifestations such as cough $\square$ fever $[$ chest pain and radiological indications of pleural lesions with or without pleural effusion.

The exclusion criteria were: presence of biopsy contraindications (low platelet count, other risks such as coagulation and bleeding, clinical instability or unsafe location of biopsy); elderly patients or patients with serious systemic diseases who could not tolerate the pleural biopsy; serum HIV positive; patients with pleural lesions can be made diagnosis do not needing biopsy or have opposite indication of pleural biopsy; patients refused to be performed by pleural biopsy; pleural lesions judged by the sonographer to be unsuitable for biopsy.

When clinically necessary and safe, we performed CEUS-guided pleural biopsy. Pleural tissue was evaluated by histopathology and etiological tests. 
Patients with pleural effusion received ultrasound-guided pleural catheter drainage, and pleural effusion was tested accordingly, including routine pleural effusion $\square$ biochemical and ADA $\square$ microbiological tests and cytological examinations.

\section{Diagnostic criteria and establishment of the final diagnosis $\square$}

Diagnosis of pleural TB: We used a composite reference standard (CRS) as the diagnosis standard. CRS included both definite and possible cases. Definite cases: Etiology positive by either MTB culture or Xpert MTB/RIF positive on pleural effusion or pleural biopsy; Possible cases: patients with TB imaging characteristics and clinical manifestations or positive TB immunological test, or histopathology confirmed as tuberculosis lesions on pleural biopsy, or biochemical examination of pleural effusion suggested TB and patients were responsive to anti-TB treatment (10).

Diagnosis of other pleural infectious diseases $₫$ Specific pathogens were identified in pleural effusion or pleural biopsy by microbiological, molecular biological, or histopathological staining and appropriate antibiotic treatment was effective.

Diagnosis of malignant pleural lesions: definitive cytological or histological examination of tumor cells.

All patients were treated and followed up for at least 6 months. Thoracoscopy was performed in patients who cannot been diagnosed after all above examinations failed to have clear diagnosis. A nonspecific diagnosis of pleurisy must be established in those with no clinical or radiological progression during follow-up $₫ 5)$.

\section{Instruments and methods [}

Ultrasound instrument LOGIQ E9 ultrasonic diagnostic instrument from GE, USA, with convex array probe, frequency $1 \sim 6 \mathrm{MHz}$. The instrument has harmonic contrast imaging function with low mechanical index (MI).

For patients with pleural effusion, pleural fluid was collected by closed thoracic drainage guided by ultrasound. CEUS was then used to find the biopsy target site.

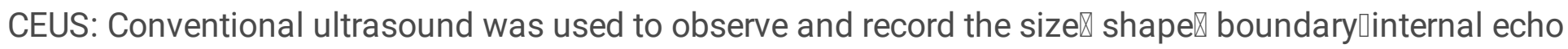
and periphery of the lesions. All patients signed the informed consent before the angiography. The instrument MI was adjusted to 0.10 and the contrast gain was $20 \mathrm{~dB} .1 .5 \mathrm{~mL}$ SonoVue ultrasound contrast agent (Bracco, Italy) was injected through the elbow vein. The dual-contrast interface was adopted. At the same time of the injection of contrast agent, the ultrasonic instrument was started with a built-in chronograph for 3 min continuous observation, and the images were stored and analyzed.

CEUS-guided biopsy: ultrasound-guided biopsy was performed immediately after CEUS. Before the biopsy, two observers evaluated the enhancement pattern. They reached a consensus that emphasized the identification of viable target areas. $2 \%$ lidocaine hydrochloride was subjected to local layer. When the 
needle tip reached the leading edge of the enhancement area of the lesion 2 3 $\mathrm{mm}$, the max-core automatic biopsy needle (model $18 \mathrm{G} \times 10 \mathrm{~cm}$ or $16 \mathrm{G} \times 10 \mathrm{~cm}$, BD Company, USA) was excited to complete a biopsy. Repeat the operation to obtain $3 \sim 4$ more complete tissue strips is appropriate冈Fig1-2).

\section{Microbiological tests:}

The pleural tissue / pleural effusion was finely ground and suspended in $1 \mathrm{ml}$ sterile saline, the pleural fluid was centrifuged (3000RPM, $15 \mathrm{~min}$ ), the supernatant was discarded, and the precipitation solution was used for further microbiological tests (Bacteria冈fungi culture区, all procedures were performed according to the protocols.

BACTECTM MGIT 960 culture (MGIT 960, BD, USA) and Gene Xpert MTB/RIF (Xpert) were used for identification of mycobacterium, including mycobacterium tuberculosis (MTB) and non-Mycobacterium tuberculosis (NTM).

\section{Pathological examination:}

The biopsy specimens were fixed with $10 \%$ neutral formalin. All formalin fixation and paraffin embedding (FFPE) sections stained with hematoxylin-eosin (HE) were observed by two experienced pathologists. Specific stain and immunohistochemistry $(\mathrm{IHC})$ were performed if needed.

\section{Complications}

Complications were recorded and classified into four categories: no, self-limited pneumothorax or bleeding, pneumothorax or bleeding requiring a chest tube and hospitalization, and other major complications (death, prolonged hospitalization, etc.).

\section{Statistical Analysis}

All data were set up in Excel 2010 and analyzed statistically with SPSS 20.0. The measurement data was compared by Student's $t$ test. Enumeration data was compared by chi-square test. Mcnemar $\chi 2$ test was used to compare the diagnostic yield of CEUS-guided sampling. A $p$-value of $<0.05$ was considered statistically significant

\section{Result}

\section{Clinical characteristics of participants $\square$}

A total of 472 patients were prospectively included from Nov 1, 2018 to Oct 31, 2019, 12 cases were excluded due to incomplete clinical data and default of follow-up. All clinical data were shown in Table 1. The flow diagram was shown in Fig3

Prior conventional ultrasound showed intra-lesion in 32.17\% (148/460) cases and peripheral obvious vessels in $21.30 \%$ ( 98/460) cases, while CEUS presented intra-lesion necrosis in 72.17\% (332/460区 
cases and obvious peripheral vessels in 55.43\% (255/460冈cases of the 460 cases enrolled, Both of which showed significant differences. $(P 0.00)$ Intra-lesion necrosis was more likely to occur in benign lesions ( $P 0.00 \rrbracket$ and intra-lesion obvious vessels were more likely to occur in malignant lesions ( $P$ 0.00》 (fig1-2】 Table 1).

\section{Overall diagnostic efficiency of CEUS-guided pleural biopsy sampling for pleural lesions:}

Among 460 cases enrolled $\mathbb{4} 44$ cases obtained diagnosis through CEUS-guided biopsy directly, including 313 cases of infectious disease (280 cases of pleural tuberculosis, 10 case of NTM , 23 cases of empyema) and 131 cases of non-infectious disease (129 cases of primary/metastatic pleural malignancy, 2 cases of mesothelioma ) , 16 cases with undefined diagnosis were underwent thoracoscopy, then $100 \%$ (16/16) of them got the definite diagnosis of all non-infectious disease (5 cases of mesothelioma , 11 cases of nonspecific pleurisy). Therefore, thoracoscopy identified 5 additional malignant lesions on the base of CEUS-guided biopsy did.

It was worth mentioned that among the 16 cases with undefined diagnosis through CEUS guided biopsy, histopathology indicated malignant lesions in 3 cases, but the specific pathological type could not be determined, which were finally diagnosed as pleural mesothelioma through thoracoscopic surgery sampling.

In 11 cases with a final diagnosis of nonspecific pleurisy, CEUS-guided biopsies obtained the same results as thoracoscopy sampling, and all of these patients were followed up for six months with stable clinical and radiological results.

Thus, we summarized the overall diagnostic accuracy of CEUS-guided pleural biopsy was $98.91 \%$ (455/460): 100\% (313/313) of infectious pleural lesions and $96.60 \%(142 / 147)$ of non-infectious pleural lesions.

Furthermore, in 330 cases of pleural lesions combined pleural effusion, CEUS-guided biopsy increased the diagnostic yield from $60.30 \%(199 / 330)$ to $98.36 \%$ (325/330冈P0.00) in all cases, from 15.56 $\% \otimes 14 / 90)$ to $\% 94.44 \%(85 / 90)$ in malignant pleural lesions ( $P 0.00 \rrbracket a n d$ from $77.08 \% \otimes 185 / 240 \otimes$ to $100 \% \otimes 240 / 240, P 0.00)$ in infectious pleural lesions.

\section{Diagnostic efficiency of CEUS-guided pleural biopsy sampling in infectious lesions:}

313 cases of infectious pleural lesions (280 cases of pleural tuberculosis, 10 case of NTM, 23 cases of empyema) were diagnosed through CEUS-guided biopsy. The diagnostic yield of CEUS-guided biopsy in infectious pleural lesions was 100\% (313/313), meanwhile the etiology detection rate was $88.82 \%$ $(278 / 313)$.

In 240 cases with pleural lesions and pleural effusion (210 cases of pleural tuberculosis, 7 case of NTM, 23 cases of empyema), CEUS-guided biopsy obtained etiological diagnosis in 205 cases (85.42\%, 
205/240), which showed significant higher than that of pleural effusion $(12.08 \%, 29 / 240, P 0.00)$. (Table 2)

In 280 cases of pleural tuberculosis, 100\% (280/280) were diagnosed through CEUS-guided biopsy using CRS as the reference standard. The etiology detection rate of CEUS-guided biopsy in pleural tuberculosis was $87.50 \%$ (245/280). In 210 cases pleural tuberculosis combined pleural effusion, CEUS-guided biopsy increased the diagnostic yield from $95.24 \%(200 / 210)$ to $100 \%(210 / 210, P 0.78)$ when using the CRS as the reference standard, meanwhile increased the etiology detection rate from $11.90 \%(25 / 210)$ to $85.24 \%$ $(179 / 210, P 0.00)$.

\section{Diagnostic efficiency of CEUS-guided pleural biopsy sampling in non-infectious lesions:}

In 147 cases with non-infectious pleural lesions, 142 cases (129 of primary/metastatic pleural malignancy, 2 of mesothelioma, 11 cases of nonspecific pleurisy) were diagnosed through CEUS-guided biopsy. The diagnostic yield of CEUS-guided biopsy in non-infectious pleural lesions were $96.60 \%$ $(142 / 147)$.

In 136 cases of malignant lesions, 131 cases (129 of primary/metastatic pleural malignancy, 2 of mesothelioma) were diagnosed through CEUS-guided biopsy. The diagnostic yield of CEUS-guided biopsy in malignant lesions were $96.32 \%$ 131/136).

In 90 cases non-infectious pleural lesions with pleural effusion, CEUS-guided biopsy obtained histopathological diagnosis in $94.44 \% \otimes 85 / 90$ ) cases, which showed significant higher than that of pleural effusion $(15.56 \%, 14 / 90, P 0.00)$ (Table 3$)$.

In 90 cases of malignant pleural lesions with pleural effusion, CEUS-guided biopsy increased the diagnostic yield from $15.56 \% \bigotimes 14 / 90)$ to $\% 94.44 \%(85 / 90)$.

\section{Occurrence of adverse reactions}

All patients underwent CEUS and USCB. During the sampling process, 23 patients experienced minor complications. The incidence of pneumothorax was $1.1 \%$ (5/460), and most of them were small pneumothorax (compression $<15 \%$ ). Only 1 patient with a history of emphysema underwent closed drainage due to pneumothorax compression of $40 \%$. The incidence of local bleeding or sputum blood was $3.91 \%(18 / 460)$ and all of them got improved after short-term local pressure, and no serious adverse events occurred.

\section{Discussion}

According to the published studies, the sensitivity of thoracoscopy for pleural lesions was $90 \%-95 \%$ (11). However, thoracoscopy is expensive, involves general anesthesia and invasive. Previous study showed that ultrasound-guided biopsies increased the combined yield of all diagnoses from $48.0 \%$ to $90.0 \%$, malignancies from $31.0 \%$ to $89.7 \%$ and tuberculosis from $77.8 \%$ to $88.9 \%$ (9). Another study of 
ultrasound-guided biopsy showed that $94 \%$ patients had sufficient tissue for histological diagnosis, and only one false-negative biopsy (2\%) was found during the median follow-up period of 16 months (12). As a minimally invasive method, CEUS-guided biopsy was used in the present study to locate the appropriated site and obtain valuable specimens. The overall diagnostic accuracy was $98.91 \%$ (455/460): 100\% (313/313) in infectious pleural lesions and 96.59\% (142/147) in non-infectious pleural lesions. In 330 cases of pleural lesions combined pleural effusion, CEUS-guided biopsy increased the diagnostic yield from $60.30 \%$ (199/330) to $98.36 \%$ (325/330هP0.00) in all cases, from $15.56 \%$ $(14 / 90)$ to $\% 94.44 \%(85 / 90)$ in malignant pleural lesions ( $P 0.00$ \and from $77.08 \% \bigotimes 185 / 240 \bigotimes$ to $100 \% \bigotimes 240 / 240, P 0.00)$ in infectious pleural lesions. After 12 months of follow-up, no false negative cases were found. The results suggested that CEUS guided biopsy may be superior to traditional ultrasound-guided biopsy, even as high as that of thoracoscopy reported (11). After CEUS, the location and adjacency of the unenhanced and enhanced areas in the lesion can be determined. Necrotic tissue and vessel can be clearly distinguished after injection of contrast agents which were favor for biopsy and improving the quality of aspiration tissure (Fig1). In the present study, CEUS presented internal necrosis in

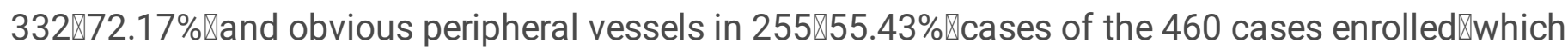
suggested that CEUS may choose a more effective and safety biopsy path than conventional ultrasonic did.

Routine treatment on pleural infectious lesions focuses on appropriate antibiotic selection and adequate drainage of pleural fluid or clear up infective lesions from the chest, closed thoracic drainage is the most common method for obtaining pleural effusion (13). Existing studies had shown that microbes are more likely to be located on the surface of the pleural wall than to "float" in the pleural fluid which is known to be acidic, hypoxic and nutrient-poor (14). Studies showed that the etiological detection rate on pleural biopsy tissue was much higher than that on pleural fluid (1区15), which was able to further guide accurate antibiotics selection. In the present study, 100\% (313/313) infectious pleural lesions were diagnosed by CEUS guided biopsy and $88.82 \%$ (278/313) obtained the etiological evidence. In 240 cases with pleural effusion, CEUS guided biopsy significantly improved the etiological diagnostic rate compared with pleural fluid extraction $(P 0.00)$. Our results demonstrated that infectious diseases should be firstly diagnosed accurately through early biopsies and confirm the etiology so as to avoid of empirical antibiotics abuse. The results was consistent with existing research (14).

In pleural TB, detection of MTB in pleural tissue culture was significantly higher than that in pleural liquid culture $(<10 \%)(15)$. In $25 \%$ of patients, pleural tissue may be the only specimen to capture the organism (16). Previous study shown that in areas with high TB burdens, ultrasound-guided biopsy has a diagnostic rate of nearly $90 \%$ and a low incidence of complications (3). In the present study, $100 \%$ (280/280) pleural TB were diagnosed through CEUS guided biopsy, and $87.5 \%(245 / 280)$ of them were diagnosed etiologically. In patients with pleural effusion, although biopsy did not significantly increase the clinical diagnostic yield $(P 0.78)$, it significantly increased the etiological diagnosis rate $(P 0.00)$. China is a country with high TB burden. There is a particular need to identify TB or drug-resistant TB through acquisition of pathogenic bacteria. The results of the study suggested that for pleural TB, 
especially "dry" pleural TB, pleural biopsy guided by CEUS can provide rapid etiological evidence at an early stage.

Of the 136 malignant lesions, $131 \otimes 96.32 \%$ هcases were confirmed by CEUS-guided biopsy. CEUS-guided biopsy was also performed on 90 cases of malignant pleural lesions with pleural effusion, and the diagnostic rate was increased from $15.56 \%(14 / 90)$ to \% $94.44 \%(85 / 90)$. Only 5 cases of mesothelioma were finally confirmed by surgical thoracoscopy. Pleural mesothelioma is a malignant pleural disease difficult to be diagnosed by minimally invasive means. Even video-assisted multipoint thoracoscopic biopsies have a certain rate of missed diagnosis (17). In the present study, $28.57 \% \varangle 2$ /7囚of mesothelioma were diagnosed by CEUS guided biopsy. It is worth noting that even among the 5 cases of mesothelioma that were not clearly diagnosed by CEUS-guided biopsy, 3 of them showed pathologically malignant lesions.

In addition, the advantage of CEUS-guided biopsy is that the entire needle biopsy process is performed under direct vision. It allows real-time observation of lung movements and allows patients to hold their breath and perform puncture immediately to avoid injury. In the present study, the incidence of complications was only $5 \%(23 / 460)$, all of which were mild and relieved after short-term symptomatic treatment. The pneumothorax occurred in only $1.1 \%$ (5/460), most of them were recovered by oxygen inhalation. Our results indicated that CEUS biopsy provides a safe diagnostic approach.

Our study had the following limitations: It did not set common ultrasound guided biopsy without contrastenhanced agents as control. Because contrast agents injected can greatly improve the differentiation of necrotic and vascular areas (fig1-2), all included patients were performed by CEUS guided biopsy under safety and ethical consideration.

In conclusion, by identifying necrosis and surrounding blood vessels, CEUS guided a more effective biopsy that was proved to be safe and minimally invasive. Highly efficient diagnostic yield could be achieved through the sampling of CEUS with high safety for pleural lesions. It was considered to be applied as an effective diagnostic method for patients with pleural lesions, especially in resource-poor areas.

\section{Declarations}

\section{Competing interests}

The authors declare that they have no competing interests.

\section{Acknowledgements}

We thank all participants for their time and efforts.

\section{Date available statement}


All data regarding the included participants and laboratory data during the study are available from the corresponding author by email request. The clinical study was registered at The China Clinical Trial Registry (ChiCTR, www.chictr.org.cn) with the registration number: ChiCTR2000029749.

\section{Author contribution Statement}

WS: included the patients, treated and followed up the patients, wrote the manuscript;

YZ: screening patients, surgical thoracoscopy; YZ and WS :Included the patients, collected the data; ZD: histopathological examination;YW and $\mathrm{CY}$ :Contrast-enhanced ultrasound and ultrasound-guided biopsy $\mathbb{Z}$ LF and ZZ: Research design, included the patients, data collection and revised the manuscript. All authors read and approved the final submitted version.

\section{Funding acknowledgements}

This work was supported by the grant from the Shanghai Natural Science Foundation (Grant No.20ZR1446700). The funders had no role in study design, data collection and analysis, decision to publish, or preparation of the manuscript.

\section{Ethics declaration and consent to participate}

This prospective study was approved by The Ethics Committee of the Shanghai Pulmonary Hospital (approval number: K18-170). Each participant or their legal representatives gave written informed consent before enrollment.

\section{Consent for publication section}

Not applicable

\section{References}

1. Bhatnagar Rahul,Corcoran John P, Maldonado Fabien et al. Advanced medical interventions in pleural disease., 2016, 25: 199-213.

2. Dixon G, de Fonseka D, Maskell N. Pleural controversies: image guided biopsy vs. thoracoscopy for undiagnosed pleural effusions? J Thorac Dis 2015;7:1041-51

3. Koegelenberg CFN, Irusen E M , Von Groote-Bidlingmaier F , et al. The utility of ultrasound-guided thoracentesis and pleural biopsy in undiagnosed pleural exudates. Thorax, 2015:995-7.

4. Khosla, R., McLean, A. W., Smith, J. A. (2016). Ultrasound-guided versus computed tomography-scanguided biopsy of pleural-based lung lesions. Lung India 33, 487-492.

5. Koegelenberg CF, Irusen EM, von Groote-Bidlingmaier F, et al. The utility of ultrasound-guided thoracentesis and pleural biopsy in undiagnosed pleural exudates. Thorax 2015; 70: 995-997.

6. Wang, S., Yang, W.,et al.The role of contrast-enhanced ultrasound in selection indication and improving diagnosis for transthoracic biopsy in peripheral pulmonary and mediastinal lesions. 
Biomed. Res. Int. 2015, 231782.

7. Dudau C, Hameed S, Gibson D, et al. Can Contrast-Enhanced Ultrasound Distinguish Malignant from Reactive Lymph Nodes in Patients with Head and Neck Cancers?.Ultrasound Med Biol, 2014, 40(4):747-754.

8. Fu Ying,Zhang Yuan-Yuan,Cui Li-Gang et al. Ultrasound-Guided Biopsy of Pleural-Based Pulmonary Lesions by Injection of Contrast-Enhancing Drugs .Front Pharmacol, 2019, 10: 960.

9. Sun Wenwen,Gu Jin,Bi Ke et al. Clinical performance of Xpert MTB/RIF on contrast-enhanced ultrasound-guided core biopsy specimens for rapid diagnosis of superficial tuberculous lymphadenitis in high TB burden settings.[J] .Infection, 2021,

10. Meldau Richard,Randall Philippa,Pooran Anil et al. Same-Day Tools, Including Xpert Ultra and IRISATB, for Rapid Diagnosis of Pleural Tuberculosis: a Prospective Observational Study.J. Clin. Microbiol., 2019, 57 (9) e00614-19

11. Maturu VN, Dhooria S, Bal A, et al. Role of medical thoracoscopy and closed-blind pleural biopsy(CPB) in undiagnosed exudative pleural effusions: a single-center experience of 348 patients. J Bronchology Interv Pulmonol 2015;22:121-9

12. Hallifax RJ, Corcoran JP, Ahmed A, et al. Physician-based ultrasound-guided biopsy for diagnosing pleural disease. Chest. 2014;146(4):1001-1006.

13. Davies HE, Davies RJ, Davies CW. Management of pleural infection in adults: British Thoracic Society Pleural Disease Guideline 2010. Thorax 2010; 65: Suppl. 2, ii41-ii53

14. Lee Y C Gary,Fitzgerald Deirdre B,Pleural Biopsy to Capture Causative Microbe: A New Piece of the Pleural Infection Jigsaw.Chest, 2018, 154: 743-745.

15. I. Psallidas, N.I. Kanellakis, R. Bhatnager, et al.A pilot feasibility study in establishing the role of ultrasound-guided pleural biopsies in pleural infection (The AUDIO study) Chest, 154 (4) (2018), pp. 766-772

16. Wilkosz S , Edwards L A , Bielsa S, et al. Characterization of a New Mouse Model of Empyema and the Mechanisms of Pleural Invasion by Streptococcus pneumoniae. American Journal of Respiratory Cell \& Molecular Biology, 2012, 46(2):180.

17. Chirieac Lucian R,Hung Yin P,Foo Wai Chin et al. Diagnostic value of biopsy sampling in predicting histology in patients with diffuse malignant pleural mesothelioma .Cancer, 2019, 125: 4164-4171.

\section{Tables}

Table 1 clinical characteristics of patients enrolled with pleural lesions: 


\begin{tabular}{|c|c|c|c|}
\hline Clinical characteristics & $\begin{array}{l}\text { Infection } \\
(n=313)\end{array}$ & $\begin{array}{l}\text { Non-infection } \\
(n=147)\end{array}$ & $P$ \\
\hline Median age, years (range) & $31(16,80)$ & $44.6(24,78)$ & $0.01^{*}$ \\
\hline \multicolumn{4}{|l|}{ Sex n (\%) } \\
\hline Male & $215(68.69)$ & $80(54.42)$ & $0.03^{*}$ \\
\hline \multicolumn{4}{|l|}{ Clinical signs and symptoms n (\%) } \\
\hline Fever & $309(98.72)$ & $12(8.16)$ & $0.00 *$ \\
\hline emaciation & $255(81.47)$ & $112(76.19)$ & 1.42 \\
\hline chest pain & $190(60.70)$ & $120(81.63)$ & $0.00 *$ \\
\hline \multicolumn{4}{|l|}{ Chest CT imaging n (\%) } \\
\hline Pleural lesion & $313(100.00)$ & $147(100.00)$ & 5.76 \\
\hline Pleural lesion with pleural effusion & $240(67.43)$ & $90(61.22)$ & 1.09 \\
\hline \multicolumn{4}{|l|}{ CEUS performance $\mathrm{n}(\%)$} \\
\hline Appearance of necrosis & $260 \otimes 79.51 \rrbracket$ & $72 \rrbracket 54.13 \rrbracket$ & $0.00 *$ \\
\hline Appearance obvious peripheral vessels & 146囚44.65囚 & 109ه81.95》 & $0.00 *$ \\
\hline
\end{tabular}

*indicated significant differences between two groups.

Table 2 Etiological detection rate of infectious pleural lesions with pleural effusion ( $n, \%)$

\begin{tabular}{|llll|}
\hline $\mathrm{n}=240$ & $\begin{array}{l}\text { pleural tuberculosis } \\
\mathrm{n}=210\end{array}$ & $\begin{array}{l}\text { NTM } \\
\mathrm{n}=7\end{array}$ & $\begin{array}{l}\text { Empyema } \\
\mathrm{n}=23\end{array}$ \\
\hline pleural effusion & $25(11.90 \%)$ & $1(14.29)$ & $3(13.04)$ \\
\hline pleural biopsy & $179(85.24 \%)$ & $6(85.71)$ & $20(86.96)$ \\
$P$ & 0.00 & 0.00 & 0.00 \\
\hline
\end{tabular}

Table 3 Histopathologic diagnostic rate of non-infectious pleural lesions with pleural effusion (n, \%) 


\begin{tabular}{|c|c|c|c|}
\hline$n=90$ & $\begin{array}{l}\text { pleural malignancy } \\
\mathrm{n}=72\end{array}$ & $\begin{array}{l}\text { mesothelioma } \\
n=7\end{array}$ & $\begin{array}{l}\text { nonspecific pleurisy } \\
n=11\end{array}$ \\
\hline pleural effusion & $14 \bowtie 19.44)$ & $0(0.00)$ & $0(0.00)$ \\
\hline pleural biopsy & $58(100.00)$ & $2(28.57)$ & $11(100.00)$ \\
\hline$P$ & 0.00 & 0.00 & 0.00 \\
\hline
\end{tabular}

\section{Figures}
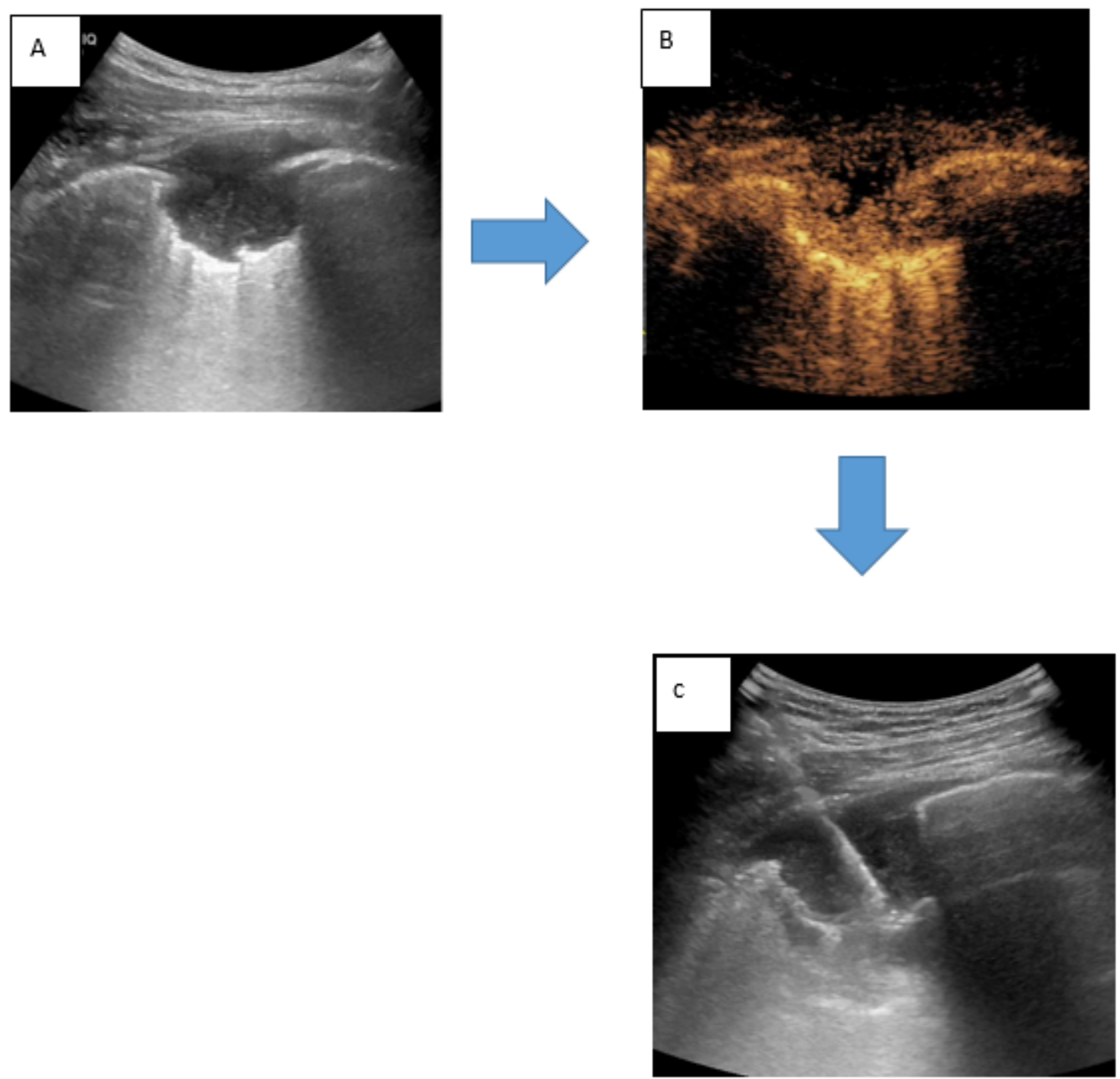

\section{Figure 1}

CEUS imaging of necrosis and CEUS guided biopsy $₫ \mathrm{~A}$ : conventional ultrasound showed the pleural lesion border discernible and internal echo evener with no obvious necrosis area. B: After CEUS, the lesion 
center performanced as contrast agents no enhancement area, thus the necrotic area could be clearly seen. C:After CEUS『ultrasound-guided percutaneous biopsy was performed, and the biopsy needle was inserted into the posterior area of the lesion
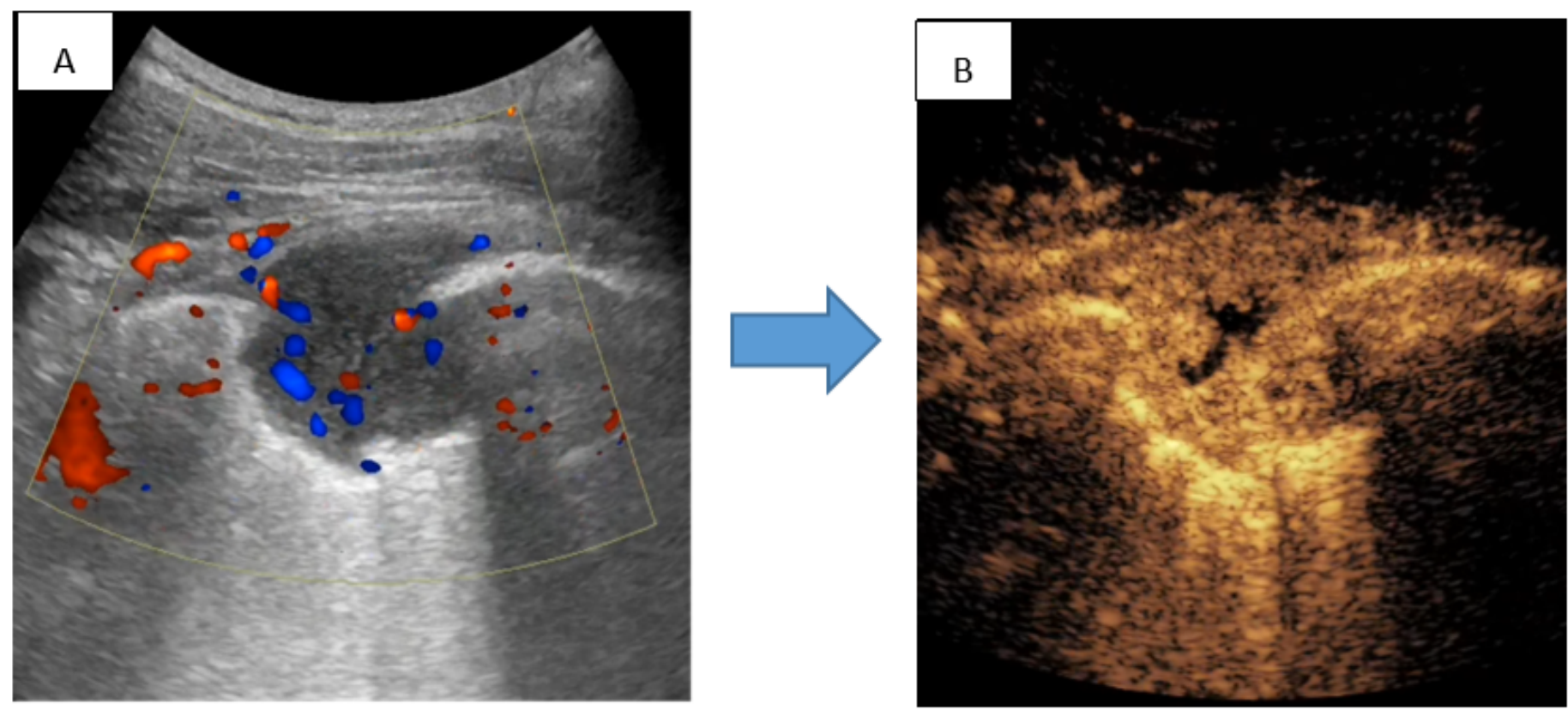

Figure 2

CEUS imaging of tumor blood vessels $\triangle \mathrm{A}$ : color doppler flow imaging (CDFI) : article point and line sample blood flow signals within lesions B: significant enhancement was observed around the lesion after CEUS , suggesting rich blood supply 『small unenhanced areas seen within the center 


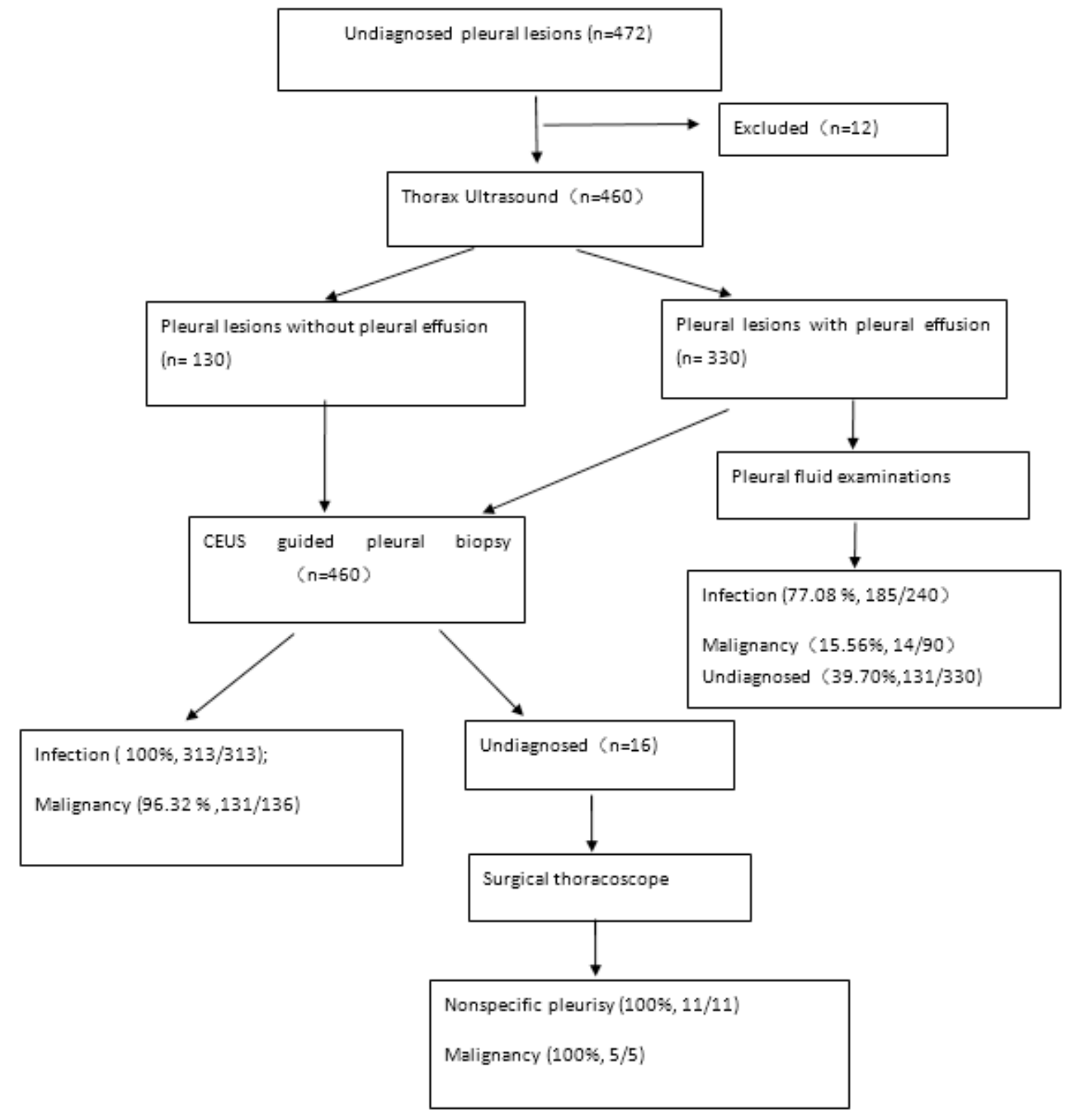

Figure 3

Flow diagram 